\title{
Copenhagen Index versus ROMA in preoperative ovarian malignancy risk stratification: result from the first Vietnamese prospective cohort study
}

\section{Doan Tu Tran}

Hue University of Medicine and Pharmacy

\section{Van Khoa Vo}

Hue University of Medicine and Pharmacy

Minh Tam Le

Hue University of Medicine and Pharmacy https://orcid.org/0000-0001-6225-3108

\section{Linus Chuang}

Nuvance Health, University of Vermont Larner College of Medicine

Vu Quoc Huy Nguyen ( $\nabla$ nvqhuy@huemed-univ.edu.vn )

Hue University of Medicine and Pharmacy https://orcid.org/0000-0002-4744-7059

\section{Research Article}

Keywords: Ovarian tumor, Copenhagen Index (CPH-I), Risk of Ovarian Malignancy Algorithm (ROMA), CA125, HE4

Posted Date: April 26th, 2021

DOl: https://doi.org/10.21203/rs.3.rs-366745/v3

License: (c) (i) This work is licensed under a Creative Commons Attribution 4.0 International License. Read Full License 
1 Copenhagen Index versus ROMA in preoperative ovarian malignancy risk 2 stratification: result from the first Vietnamese prospective cohort study

Tran Doan $\mathrm{Tu}^{1}$, Vo Van Khoa ${ }^{2}$, Le Minh Tam³, Linus Chuang ${ }^{4}$, Nguyen Vu Quoc Huy 5 , *

${ }^{1}$ M. D, MSc, Department of Obstetrics and Gynecology, Hue University of Medicine and Pharmacy, Hue University, 06 Ngo Quyen Street, Hue 491200, Vietnam

${ }^{2}$ M. D, MSc, Department of Obstetrics and Gynecology, Hue University of Medicine and Pharmacy, Hue University, 06 Ngo Quyen Street, Hue 491200, Vietnam

${ }^{3}$ M. D, PhD, Assoc. Professor, Department of Obstetrics and Gynecology, Hue University of Medicine and Pharmacy, Hue University, 06 Ngo Quyen Street, Hue 491200, Vietnam. ${ }^{4}$ MD, Professor, Obstetrics and Gynecology, Nuvance Health, University of Vermont Larner College of Medicine, United States

5 M. D, PhD, Professor, Department of Obstetrics and Gynecology, Hue University of Medicine and Pharmacy, Hue University, 06 Ngo Quyen Street, Hue 491200, Vietnam.

2306 Ngo Quyen Street,

24 Hue 491200, Vietnam

25 Phone: +84 2343822173

26 Fax: +84234 3826269

27 Email: nvqhuy@huemed-univ.edu.vn

28 ORCID 0000-0002-4744-7059 


\section{ABSTRACT}

Objectives: This study aimed to evaluate the diagnostic performances of the Copenhagen Index $(\mathrm{CPH}-\mathrm{I})$ and Risk of Ovarian Malignancy Algorithm (ROMA) in the preoperative

32 prediction of ovarian cancer.

Methods: In a prospective cohort study, data were collected from 475 patients with ovarian masses diagnosed by gynecologic examination / ultrasound who were hospitalized at the Departments of Obstetrics and Gynecology, Hue University of Medicine and Pharmacy Hospital and Hue Central Hospital, Vietnam, between January 2018 and June 2020. ROMA and $\mathrm{CPH}-\mathrm{I}$ were calculated based on measurements of serum carbohydrate antigen (CA125) and human epididymis protein (HE4). The final diagnosis was based on clinical features, radiologic and histologic findings, and the International Federation of Gynecology and Obstetrics (FIGO) 2014 stages of ovarian cancer were recorded. Matching the values of ROMA and $\mathrm{CPH}-\mathrm{I}$ to postoperative histopathology reports resulted in the preoperative prediction values.

Results: Among the 475 women, 408 had benign tumors, 5 had borderline tumors and 62 had malignant tumors. The two indices showed similar discriminatory performances with no significant differences $(p>0.05)$. At an optimal cut-off, the sensitivities/specificities of ROMA and $\mathrm{CPH}-\mathrm{I}$ for ovarian cancer diagnosis were $74.2 \%$ and $91.8 \%, 87.1 \%$ and $78.5 \%$, respectively. The optimal cut-off for $\mathrm{CPH}-\mathrm{I}$ was $1.89 \%$. The areas under the ROC curves (AUCs) of ROMA and CPH-I were 0.882 (95\% Cl: $0.849-0.909)$ and 0.898 (95\% Cl: 0.867 $-0.924)$, respectively.

Conclusions: The introduction of the Copenhagen Index to help stratify the malignancy risk of ovarian tumors, irrespective of menopausal status, might be applied as a simple alternative with a similar efficacy to ROMA in clinical practice.

Word count: 261.

Key words: Ovarian tumor, Copenhagen Index (CPH-I), Risk of Ovarian Malignancy 
55 Algorithm (ROMA), CA-125, HE4.

Page 3 of 18 
INTRODUCTION

Ovarian cancer $(\mathrm{OC})$ is one of the ten most commonly diagnosed cancers in women and has the highest mortality rate and the worst prognosis of all gynecological cancers [1]. In 2018, 295,414 cases of OC were detected worldwide, and 184,799 died, with the highest incidence in developed countries [2]. The mortality rate has not changed in the past 30 years, and it is predicted that by 2040 , this rate will be significantly increasing $[3,4]$. Since $70 \%$ of ovarian cancers are diagnosed in an advanced stage (stage III - IV), when the disease has spread to the pelvic and abdominal region, the 5-year survival rate is $20-25 \%$, but if detected in the early stage, this rate is increased to $90 \%[3,5]$. Therefore, early detection has important implications for the treatment, quality of life and prognosis of patients $[6,7]$.

In the United Kingdom Collaborative Trial of Ovarian Cancer Screening (UKCTOCS), there were 78 ovarian cancer cases in 1,590 adnexal tumors, which were detected after screening 48,230 women by transvaginal ultrasound and 50,078 women using biochemical markers (CA-125, HE4) [8]. In 2009, Moore et al. developed the Risk of Ovarian Malignancy Algorithm (ROMA) by integrating serum CA-125 and HE4 values and menopausal status to differentiate between low- and high-risk patients with OC [9]. In 2015, Karlsen et al. developed the Copenhagen Index $(\mathrm{CPH}-\mathrm{I})$ based on these two biomarkers and patient age. The areas under the ROC curve (AUCs) that predicted OC by $\mathrm{CPH}-\mathrm{I}$ and ROMA were 0.960 and 0.954 , respectively, thereby showing that the values of these two indicators were equal. The Copenhagen Index has the advantage of not depending on ultrasound and menopausal status, and the age variable is easy to collect, simple, and objective [10]. Therefore, the Copenhagen Index's advent promises to be a reliable, objective, and widely applied tool at the grassroots level. The aim of this study was to compare the Copenhagen Index and the ROMA in the preoperative prediction of ovarian cancer. 
This was a prospective cohort study conducted at the Departments of Obstetrics and

84 Gynecology, Hue University of Medicine and Pharmacy Hospital and Hue Central Hospital,

85 Vietnam, between January 2018 and June 2020.

86 The sample size was calculated according to the formula to estimate the specificity in two

87 steps:

88 Step 1: Calculate $F P+T N$

$$
F P+T N=\frac{Z_{\frac{\alpha}{2}}^{2} x p_{s p} x\left(1-p_{s p}\right)}{w^{2}}
$$

Step 2: Calculate sample size

Abbreviations: FP, False Positive; TN, True Negative. Z: the "Z" value for Confidence Interval of $95 \%\left(Z_{\frac{\alpha}{2}}^{2}=1.96\right.$ with $\left.\alpha=0.05\right) ; \mathrm{w}($ Errors $)=0.04 ; p_{\mathrm{sp}}$, the specificity from the study of Adriana Yoshida (2016) is 0.844 [11]. pdis, the prevalence rate, according to GLOBOCAN (2018), the prevalence rate of OC in Vietnam ( $\left.p_{\text {dis }}\right)$ is 7.67 cases $/ 100,000$ women $=$

0.000767 [2]. $N_{s p}$, the minimum sample size for specificity.

The calculated minimum sample size was 317 subjects. At the end of the study, 475 women who met the selection criteria were included. This number represents most women who were diagnosed and operated on for an ovarian mass during two and half years of the study 101 at two facilities.

After administrative interviews, medical history taking and physical examination, patient having pelvic mass on gynecologic examination was diagnosed with ovarian tumor by transabdominal and transvaginal ultrasonography. An adnexal lesion was described 105 according to the morphological and vascular features as suggested by the consensus 
106 opinion from the International Ovarian Tumor Analysis (IOTA) group [12]. Blood serum 107 sample were taken for CA-125 and HE4 tests. The ovarian masses were then removed 108 surgically. Complete histopathological evaluation carried out based on the standard and 109 classification of the World Health Organization (WHO), 2014 [13]. Finally, the parameters 110 were matched with the histopathological results (including benign, borderline and malignant 111 tumors) to calculate the diagnostic values of $\mathrm{CPH}-\mathrm{I}$ and ROMA.

112 At Hue University of Medicine and Pharmacy Hospital, serum CA-125 and HE4 tests were 113 conducted using an electrochemiluminescence immunoassay on the COBAS 6000 system, 114 Roche, Switzerland. Test results were controlled by the Internal Quality Control (IQC) 115 system with RANDOX's standard control samples and programs. Calibration samples were 116 performed daily on the system of testing machines before being tested. At Hue Central 117 Hospital, tests for CA-125 and HE4 were conducted by chemiluminescent microparticle 118 immunoassay on an Architect 11000 system (Abbott Diagnostics). The tests were quality 119 checked daily (internal inspection) and were subjected to external inspection at the Ho Chi 120 Minh City Test Standardization Center.

121 The Copenhagen Index predicts the risk of a preoperative ovarian tumor malignancy 122 according to the algorithm below [10]:

$$
\mathrm{CPH}-\mathrm{I}=-14,0647+1,0649{ }^{*} \log _{2}(\mathrm{HE} 4)+0,6050{ }^{*} \log _{2}(\mathrm{CA}-125)+0,2672{ }^{*} \mathrm{Age} / 10
$$

$$
\mathrm{PP}=\mathrm{e}^{(\mathrm{CPH}-\mathrm{I})} /\left(1+\mathrm{e}^{(\mathrm{CPH}-\mathrm{I})}\right)
$$

125 The ROMA index was calculated to predict the risk of ovarian tumor malignancy before 126 surgery according to the following algorithm: ROMA $(\%)=\exp (\mathrm{PI}) /[1+\exp (\mathrm{PI})]^{*} 100$ PI is the prediction index, determined as follows:

$$
\text { - Premenopausal women: } \mathrm{PI}=-12,0+2,38^{*} \operatorname{Ln}[\mathrm{HE} 4]+0,0626^{*} \operatorname{Ln}[\mathrm{CA}-125]
$$


The ROMA cut-off point values were applied according to the technical instructions of the Cobas 6000 system and the ARCHITECT system. Patients have a high risk of ovarian 132 cancer when:

\begin{tabular}{|lll|}
\hline Test system & Pre-menopausal group & Post-menopausal group \\
\hline Cobas 6000 (ROMA 1) & $\geq 11,4 \%$ & $\geq 29,9 \%$ \\
Architect i1000 (ROMA 2) & $\geq 7,4 \%$ & $\geq 25,3 \%$ \\
\hline
\end{tabular}

\section{Statistical analysis}

Data analyses were performed using the statistical software SPSS 20.0 (SPSS, Inc., Chicago, IL, USA), and receiver operative curve (ROC) analysis was performed with MedCalc. Categorical variables were reported as numbers (percentages), and continuous variables were reported as medians (SDs, standard deviations; ranges). The chi-square test $(\chi 2)$ was used to evaluate intergroup differences, and $p<0.05$ was considered significant.

The Kruskal-Wallis test was used to compare the differences between three groups that were not normally distributed.

\section{Ethical Approval}

142 Ethical approval for the study protocol was by the Ethics Committee for Biomedical 143 Research at Hue University of Medicine and Pharmacy, Hue, Vietnam (number H2018/359). 144 Informed consent was obtained from all study subjects.

\section{RESULTS}

146 Of the 475 patients, 408,5 and 62 subjects were diagnosed with benign tumors, borderline 147 tumors, and OC, respectively. The main characteristics of individual patient subgroups 148 according to histopathologic diagnosis are shown in Table 1. The mean age of women in 
149 the OC group was higher than that of women in the benign tumor group. There were 150 significant differences in age, menopausal status, and marital status between the two 151 groups $(p<0.05)$. The incidence of OC in the postmenopausal group was $59.7 \%$.

152 Histological classification of participants was demonstrated as follows: among the 408 153 women diagnosed with benign tumors, 171 (41.9\%) had mature cystic teratoma, 165 154 (40.4\%) had serous cystadenoma, and 37 (9.1\%) had endometrioses of the ovary. In the 155 156 borderline tumor group, 4 of 5 patients had serous borderline tumors. In patients with OC, serous adenocarcinoma was seen in 27 cases (43.5\%), followed by 12 (19.7\%) with 157 mucinous adenocarcinoma, $6(9.7 \%)$ with poorly differentiated carcinoma, and $6(9.7 \%)$ with 158 159 160 dysgerminoma, as shown in Table 2. Clinical staging was performed according to the International Federation of Gynecology and Obstetrics (FIGO): 19 (30.6\%) cases were stage I, 8 (12.9\%) cases were stage II, 26 (41.9\%) cases were stage III, and 9 (14.5\%) cases were stage IV.

162 The median values of $\mathrm{CPH}-\mathrm{I}$ and ROMA of the $\mathrm{OC}$ group were statistically higher than those 163 of the benign tumor group (Kruskal - Wallis test) (Table 3). In the study sample, the median 164 value of $\mathrm{CPH}-\mathrm{I}$ in the $\mathrm{OC}$ group was $24.81 \%$ (3.49-81.21\%), which was statistically higher 165 than the value from the benign tumor group at $0.82 \%(0.44-1.76 \%)(p<0.05)$. The median 166 ROMA value in the benign tumor group was 5.03\% (3.46-8.71\%) and that of the OC group 167 was $49.93 \%(12.78-81.22 \%)$; the difference was statistically significant $(p<0.05)$. The 168 median values of the $\mathrm{CPH}-\mathrm{I}$ and ROMA of the postmenopausal group were higher than 169 those of the premenopausal group, in both the OC group and the benign tumor group. 170 Specifically, in the premenopausal group, the median values of $\mathrm{CPH}-\mathrm{I}$ for the $\mathrm{OC}$ group, benign 171 tumor, and borderline tumor group were 4.87\% (1.49 - 45.72\%), 0.72\% (0.41-1.43\%) and $1720.42 \%(0.29-24.39 \%)$, respectively; the median values of ROMA for the OC group, benign 173 tumor group, and borderline tumor group were 12.18\% (6.11-62.06\%), 4.58\% (3.06-6.76\%) 174 and 5.84\% (3.28 - 16.38\%), respectively. For the postmenopausal subjects, the median values 175 of $\mathrm{CPH}-\mathrm{I}$ for the OC group and benign tumor group were $45.49 \%(8.35-91.62 \%)$ and $1.49 \%$ 
176 (0.87-3.65\%), respectively; the median values of ROMA for the OC group and benign tumor 177 group were $72.37 \%(37.41-95.16 \%)$ and $10.59 \%$ (7.49 - 18.88\%), respectively.

178 The prognostic values of the Copenhagen Index and the ROMA index in the prediction of 179 OC risk before surgery are shown in Table 4 and Graph 1. In the study population, the AUCs 180 of $\mathrm{CPH}-\mathrm{I}$ and ROMA in the prediction of OC were equivalent, being 0.898 (95\% Cl: $0.867-$ 181 182 $0.924)$ and 0.882 (95\% Cl: $0.849-0.909)$, respectively. At the optimal cut-off point of $1.89 \%$, the Copenhagen Index had a sensitivity of $87.1 \%(95 \% \mathrm{Cl}: 76.1-94.3 \%)$ and specificity of $78.5 \%$ (95\% Cl: $74.2-82.3 \%)$. With an optimal cut-off value of $16.5 \%$ for ROMA, the sensitivity and specificity were $74.2 \%(95 \% \mathrm{Cl}: 61.5$ - 84.5\%) and $91.8 \%(95 \% \mathrm{Cl}: 88.7$ $94.2 \%)$, respectively. The Copenhagen Index and the ROMA index are of equivalent value in the differential diagnosis of benign and malignant ovarian tumors; the difference was not statistically significant $(p>0.05)$.

\section{DISCUSSIONS}

The present study aimed to compare the Copenhagen Index and the ROMA in the 190 preoperative prediction of ovarian cancer. Our data showed that Copenhagen Index is similarly accurate to but simpler than ROMA to stratify the risk of ovarian tumor malignancy, 192 irrespective of menopausal status.

The rates of OC from several studies were 41.6\% (Yoshida et al., 2016) [11], 43\% (Minar et 194 al., 2018) [14], and can be up to $57.9 \%$ (Aslan et al., 2020) [15] among postmenopausal women, quite similar to those from our study, at $59.7 \%$ - higher than the rate of about $30 \%$ as stated in some medical textbook; however, the peak age incidence of invasive epithelial ovarian cancer is approximately 60 years (Berek \& Novak's Gynecology, 16th ed., 2020) [16]. In Vietnam and in many others low- and middle-income countries, women with ovarian masses were often diagnosed not early, due to the lack of systematic screening program by tumor markers or ultrasound; and the often centrally overweight status of postmenopausal 
201 women could lead to late detection of abdominal masses in those women. These facts could 202 explain the higher rate of OC among post-menopausal women found within present study.

203 The median values of $\mathrm{CPH}-\mathrm{I}$ and ROMA in the OC group, as shown in Table 3, were higher 204 than those of the benign tumor group and borderline tumor group. Compared to previous 205 studies, the median values of $\mathrm{CPH}-\mathrm{I}$ and ROMA from our research are lower than those of 206 some other studies in the world. According to Adriana Yoshida (2016), the median values 207 of $\mathrm{CPH}-\mathrm{I}$ for benign tumors and ovarian carcinomas were $1.4 \%$ and $83.4 \%$, respectively 208 [11]. Meanwhile, in Lubos Minar's study, the median values of $\mathrm{CPH}-\mathrm{I}$ in the benign and 209 malignant groups were $2.2 \%$ and $75.4 \%$, respectively [14]. More detailed analysis in the 210 premenopausal and postmenopausal groups, which examined the differences between $211 \mathrm{CPH}-\mathrm{I}$ and ROMA, showed that the median values of $\mathrm{CPH}-\mathrm{I}$ and ROMA were higher in the 212 postmenopausal group than in the premenopausal group (Table 3). The median values of $213 \mathrm{CPH}-\mathrm{I}$ and ROMA in the postmenopausal group were higher than those in the 214 premenopausal group. The sensitivity/specificity (Se/Sp) of $\mathrm{CPH}-\mathrm{I}$ in the absence of 215 marginal ovarian tumors, nonepithelial OC, and OC metastasis was $89.7 \% / 85.3 \%$, but if the 216 above objects were included, the corresponding Se/Sp became lower at 73.1\%/84.4\% [11].

217 The Se/Sp of ROMA and $\mathrm{CPH}-\mathrm{I}$ in the diagnosis of OC were $74.2 \% / 91.8 \%$ and $21887.1 \% / 78.5 \%$, respectively. The optimal cut-off point of the $\mathrm{CPH}-\mathrm{I}$ was $1.89 \%$, and the 219 AUCs of ROMA and CPH-I were $0.882(95 \% \mathrm{Cl}: 0.849-0.909)$ and 0.898 (95\% Cl: 0.867 220 - 0.924), respectively. The work by T. Nikola (2017) on differential diagnosis between 221 ovarian endometriosis and ovarian carcinoma showed that the accuracy of the 222 Copenhagen Index was higher than that of ROMA, 93.75\% and $85.42 \%$, respectively [17].

223 Wang et al. (2019) argued that the HE4 level and ROMA and CPH-I values of epithelial 224 ovarian cancer (EOC) stages I and II (I + II) were all higher than those of borderline ovarian 225 tumor (BOT) stages I + II and benign groups in all premenopausal and postmenopausal 226 groups $(p<0.01)$. When distinguishing BOT I+II from EOC I+II, the AUC-ROCs of CPH-I 
227 and HE4 were larger than that of CA-125 $(p<0.001)$. CPH-I is more valuable than CA-125 228 when distinguishing marginal ovarian tumors from stage I - II ovarian carcinoma, while 229 HE4 may be better than CA-125 in the postmenopausal group; HE4 and $\mathrm{CPH}-\mathrm{I}$ have been 230 more advantageous than CA-125 when differentiating a borderline ovarian tumor from an 231 early-stage ovarian carcinoma (I + II) in the absence of histology or type of serum fluid. 232 The AUCs of CPH-I and ROMA in the premenopausal group were 0.779 and 0.760 , 233 respectively, and those in the postmenopausal group were 0.802 and 0.774 , respectively. 234 In the premenopausal group, the Se/Sp of ROMA and CPH-I were $78.69 \% / 64.75 \%$ and $23570.49 \% / 78.69 \%$, respectively. In the postmenopausal group, the Se/Sp of ROMA and $236 \mathrm{CPH}-\mathrm{I}$ were $82.98 \% / 68.18 \%$ and $85.11 \% / 68.18 \%$, respectively [18].

237 According to Høgdall (2016), ROMA and CPH-I can be used for the differential diagnosis 238 between benign and malignant ovarian tumors [19]. Since family doctors might be unable 239 to perform an abdominal ultrasound test, both ROMA and $\mathrm{CPH}-\mathrm{I}$ could provide the initial 240 reliable information, which helps the patient obtain early diagnosis and proper treatment 241 from specialized centers. In general, $\mathrm{CPH}-\mathrm{I}$ and ROMA have similar sensitivity and 242 accuracy. $\mathrm{CPH}-\mathrm{I}$ is not identical to ROMA and RMI because it is independent of ultrasound 243 test and menopausal status. Menopausal status can be determined based on age, 244 hormone concentration or amenorrhea per year, so the diagnosis of menopausal status 245 has not been standardized. Therefore, CHP-I could be a simpler method to optimize 246 management when assessing women with suspected OC, including age instead of 247 menopausal status $[10,19]$.

$248 \quad$ Table 5

249 Diagnostic validity of $\mathrm{CPH}-\mathrm{I}$ and ROMA from literature.

\begin{tabular}{|lllll|}
\hline \multirow{2}{*}{ Authors } & \multicolumn{2}{l}{ Copenhagen Index } & \multicolumn{2}{l|}{ ROMA } \\
\cline { 2 - 5 } & AUC & Se/Sp (\%) & AUC & Se/Sp (\%) \\
\hline A. Yoshida (2016) [11] & 0.84 & $73.1 / 84.4$ & 0.82 & $71.2 / 83.5$ \\
\hline
\end{tabular}




\begin{tabular}{|lllll|}
\hline L. Minar (2017) [14] & 0.81 & $69.0 / 85.0$ & 0.83 & $71.0 / 88.0$ \\
T. Nikolova (2017) [17] & 0.91 & $81.8 / 97.3$ & 0.90 & $90.9 / 83.8$ \\
Z. Wang (2019) [18] & 0.810 & $78.7 / 74.3$ & 0.807 & $62.9 / 88.2$ \\
Estrid Høgdall (2016) [19] & 0.960 & - & 0.954 & - \\
$\begin{array}{l}\text { Nguyen Vu Quoc Huy (2018) } \\
\text { [20] }\end{array}$ & - & - & 0.912 & $86.7 / 88.7$ \\
This study & $\mathbf{0 . 8 9 8}$ & $\mathbf{8 7 . 1 / 7 8 . 5}$ & $\mathbf{0 . 8 8 2}$ & $\mathbf{7 4 . 2 / 9 1 . 8}$ \\
\hline
\end{tabular}

250 Over 25 years ago, Jacobs et al proposed an algorithm, the Risk of Malignancy Index (RMI), 251 by combining the values of CA125, ultrasound, and menopause [21]. In 2016, Meys et al. 252 conducted a meta-analysis based on 47 articles (from January 1990 to August 2015), 253 enrolling 19,674 adnexal tumors, the Se and Sp of RMI were 0.75 (95\% Cl: 0.72-0.79) and 2540.92 (95\% Cl: 0.88-0.94), respectively [22]. According to Karlsen et al., AUCs of CPH-I, 255 ROMA and RMI were $0.960,0.954$ and 0.959 respectively in the training study and 0.951 , 256 0.953 and 0.935 respectively in the validation study. Using a Se of $95 \%$, the Sp for $\mathrm{CPH}-\mathrm{I}$, 258 the validation cohort $67.3 \%, 70.7 \%$ and $69.5 \%$ respectively [10]. This suggests that all of 259 these simple indicators, especially the RMI and the $\mathrm{CPH}-\mathrm{I}$, were of clinical significance for 260 stratifying the risk of ovarian tumor malignancy.

261 The Copenhagen Index is a new indicator that has been introduced in several studies 262 around the world. The ROMA algorithm is an index that the US Food and Drug 263 Administration has introduced in clinical practice to distinguish benign and malignant ovarian 264 tumors based on three variables: CA-125, serum HE4, and menopausal status [23]. These 265 two indexes have quite similar values since both are partially based on CA-125 and HE4. 266 Since serum CA-125 and HE4 concentrations are affected by many factors, including age, 267 smoking, uterine fibroids, pregnancy, endometriosis, pelvic inflammatory disease, and 268 gallbladder stones, this will affect the values of the Copenhagen index and ROMA [24,25]. 269 In the future, more research on these two indicators on different target groups should be 
270 conducted to clarify these differences, aiming to overcome the limitations of these indicators

271 and improve clinical practice.

272 To the best of our knowledge, this is the first prospective cohort study from Vietnam with 273 large number of ovarian tumor subjects included, examining the validity of $\mathrm{CPH}-\mathrm{I}$ and 274 comparing it with those from ROMA in risk stratification for ovarian tumor malignancy. 275 Although rigorously designed and implemented, limitations of this study included the limited 276 number of $\mathrm{OC}$ cases; and the laboratory equipments were different at the two facilities where 277 the work was done, which could partially affect the homogeneity of the data analysis. 278 Another limitation of the present study was the value of $\mathrm{CPH}-\mathrm{I}$ of the borderline tumors group 279 was even lower than that of benign tumor $(0.42 \%$ vs. $0.82 \%)$, due to the limited number of 280 borderline tumors - to be separately analyzed and to have statistical power. To overcome 281 this limitation, we combined borderline ones into the non-OC group and to compare with OC 282 group.

\section{CONCLUSIONS}

284 The introduction of the Copenhagen Index to help stratify the risk of ovarian tumor 285 malignancy, irrespective of menopausal status, is similarly accurate to but simpler than 286 ROMA and could therefore replace ROMA in clinical practice.

288 Author contributions

289 TDT, LMT, LC, and NVQH conceived the study, coordinated its planning and 290 implementation, and wrote the manuscript.

291 TDT, VVK, LMT coordinated data acquisition, participated in the data analysis and 292 interpreted the results

293 LMT, LC and NVQH supervised the preparation and revision of the manuscript.

294 All authors have approved the submitted version of the manuscript. 


\section{Acknowledgment}

296 The authors would like to thank all women who agreed to participate to this study.

\section{Conflicts of interest}

298 The authors have no conflicts of interest.

299 Funding

300 Not applicable.

\section{Author information}

303 Tran Doan Tu: MD, MSc, Department of Obstetrics \& Gynecology, Hue University of 304 Medicine and Pharmacy, Hue University, Hue 49120, Vietnam

305 Vo Van Khoa: MD, MSc, Department of Obstetrics \& Gynecology, Hue University of 306 Medicine and Pharmacy, Hue University, Hue 49120, Vietnam

307 Le Minh Tam: M.D, PhD, Assoc. Professor, Department of Obstetrics and Gynecology, Hue 308 University of Medicine and Pharmacy, Hue University, 06 Ngo Quyen Street, Hue 491200, 309 Vietnam

310 Linus Chuang: MD, Professor, Obstetrics and Gynecology, Nuvance Health, University of 311 Vermont Larner College of Medicine, United States

312 Nguyen Vu Quoc Huy: Professor, MD, PhD, Department of Obstetrics \& Gynecology, Hue 313 University of Medicine and Pharmacy, Hue University, Hue 49120, Vietnam 


\section{References}

317 [1] Z. Momenimovahed, A. Tiznobaik, S. Taheri, H. Salehiniya, Ovarian cancer in the world: epidemiology and risk factors, Int J Womens Health. 11 (2019) 287-299.

[2] F. Bray, J. Ferlay, I. Soerjomataram, R.L. Siegel, L.A. Torre, A. Jemal, Global cancer statistics 2018: GLOBOCAN estimates of incidence and mortality worldwide for 36 cancers in 185 countries, CA Cancer J Clin. 68 (2018) 394-424.

[3] S. Jacinta, Ovarian Cancer Biomarkers: Moving Forward in Early, in: Tumor Microenvironment, Springer Nature Switzerland AG. (2020) 356-358.

[4] World Ovarian Cancer Coalition, The World ovarian cancer coalition atlas. Global trends in incidence, mortality and survival. (2018) 4-32.

[5] R. Casanova, A. Chuang, Ovarian and Adnexal Disease, in: Beckmann and Ling's Obstetrics and Gynecology 8th, American College of Obstetricians and Gynecologists. (2019) 1017-1019.

[6] B. Kim, Y. Park, B. Kim, H.J. Ahn, K.-A. Lee, J.E. Chung, S.W. Han, Diagnostic performance of CA 125, HE4, and risk of Ovarian Malignancy Algorithm for ovarian cancer, J Clin Lab Anal. 33 (2019) 22624-22632.

[7] J.C. Schink, Current initial therapy of stage III and IV ovarian cancer: challenges for managed care, Semin Oncol. 26 (1999) 2-7.

[8] A. Gentry-Maharaj, M. Burnell, J. Dilley, A. Ryan, C. Karpinskyj, R. Gunu, S. Mallett, J. Deeks, S. Campbell, I. Jacobs, S. Sundar, U. Menon, Serum HE4 and diagnosis of ovarian cancer in postmenopausal women with adnexal masses, American Journal of Obstetrics and Gynecology. 222 (2020) 56.e1-56.e17.

[9] R.G. Moore, D.S. McMeekin, A.K. Brown, P. DiSilvestro, M.C. Miller, W.J. Allard, W. Gajewski, R. Kurman, R.C. Bast, S.J. Skates, A novel multiple marker bioassay utilizing HE4 and CA125 for the prediction of ovarian cancer in patients with a pelvic mass, Gynecologic Oncology. 112 (2009) 40-46.

[10] M.A. Karlsen, E.V.S. Høgdall, I.J. Christensen, C. Borgfeldt, G. Kalapotharakos, L. Zdrazilova-Dubska, J. Chovanec, C.A.R. Lok, A. Stiekema, I. Mutz-Dehbalaie, A.N. Rosenthal, E.K. Moore, B.A. Schodin, W.W. Sumpaico, K. Sundfeldt, B. Kristjansdottir, I. Zapardiel, C.K. Høgdall, A novel diagnostic index combining HE4, CA125 and age may improve triage of women with suspected ovarian cancer - An international multicenter study in women with an ovarian mass, Gynecol. Oncol. 138 (2015) 640646.

[11] A. Yoshida, S.F. Derchain, D.R. Pitta, L.A.L. De Angelo Andrade, L.O. Sarian, Comparing the Copenhagen Index $(\mathrm{CPH}-\mathrm{I})$ and Risk of Ovarian Malignancy Algorithm (ROMA): Two equivalent ways to differentiate malignant from benign ovarian tumors before surgery?, Gynecologic Oncology. 140 (2016) 481-485. 
[12] D. Timmerman, L. Valentin, T.H. Bourne, W.P. Collins, H. Verrelst, I. Vergote, International Ovarian Tumor Analysis (IOTA) Group, Terms, definitions and measurements to describe the sonographic features of adnexal tumors: a consensus opinion from the International Ovarian Tumor Analysis (IOTA) Group, Ultrasound Obstet Gynecol. 16 (2000) 500-505.

[13] V. Dochez, H. Caillon, E. Vaucel, J. Dimet, N. Winer, G. Ducarme, Biomarkers and algorithms for diagnosis of ovarian cancer: CA125, HE4, RMI and ROMA, a review, J Ovarian Res. 12 (2019) 28-37.

[14] L. Minar, M. Felsinger, Z. Cermakova, F. Zlamal, J. Bienertova-Vasku, Comparison of the Copenhagen Index versus ROMA for the preoperative assessment of women with ovarian tumors, Int J Gynecol Obstet. 140 (2018) 241-246.

[15] K. Aslan, MA. Onan, C. Yilmaz, N. Bukan, M. Erdem, Comparison of HE 4, CA 125, ROMA score and ultrasound score in the differential diagnosis of ovarian masses., J Gynecol Obstet Hum Reprod. 49 (2020) 101713-101713.

[16] J.S. Berek, E. Novak, eds., Ovarian, Fallopian Tube, and Peritoneal Cancer, in: Berek \& Novak's Gynecology, 15th ed, Wolters Kluwer Health/Lippincott Williams \& Wilkins, Philadelphia. 37 (2020) 2337-2339.

[17] T. Nikolova, R. Zivadinovic, N. Evtimovska, V. Klisarovska, M. Stanojevic, J. Georgievska, N. Nikolova, Diagnostic performance of human epididymis protein 4 compared to a combination of biophysical and biochemical markers to differentiate ovarian endometriosis from epithelial ovarian cancer in premenopausal women, J. Obstet. Gynaecol. Res. 43 (2017) 1870-1879.

[18] Z. Wang, X. Tao, C. Ying, CPH-I and HE4 Are More Favorable Than CA125 in Differentiating Borderline Ovarian Tumors from Epithelial Ovarian Cancer at Early Stages, Disease Markers. 2019 (2019) 1-10.

[19] E. Høgdall, Approaches to the detection of ovarian cancer, Scandinavian Journal of Clinical and Laboratory Investigation. 76 (2016) 49-53.

[20] N.V.Q. Huy, V. Van Khoa, L.M. Tam, T.Q. Vinh, N.S. Tung, C.N. Thanh, L. Chuang, Standard and optimal cut-off values of serum ca-125, HE4 and ROMA in preoperative prediction of ovarian cancer in Vietnam, Gynecologic Oncology Reports. 25 (2018) 110-114.

[21] I. Jacobs, D. Oram, J. Fairbanks, J. Turner, C. Frost, J.G. Grudzinskas, A risk of malignancy index incorporating CA 125, ultrasound and menopausal status for the accurate preoperative diagnosis of ovarian cancer, Br J Obstet Gynaecol. 97 (1990) 922-929.

[22] E.M.J. Meys, J. Kaijser, R.F.P.M. Kruitwagen, B.F.M. Slangen, B. Van Calster, B. Aertgeerts, J.Y. Verbakel, D. Timmerman, T. Van Gorp, Subjective assessment versus 
ultrasound models to diagnose ovarian cancer: A systematic review and meta-analysis, Eur J Cancer. 58 (2016) 17-29.

392 [23] R.G. Moore, M.C. Miller, P. Disilvestro, L.M. Landrum, W. Gajewski, J.J. Ball, S.J.

[25] S. Kumari, Serum Biomarker Based Algorithms in Diagnosis of Ovarian Cancer: A Review, Indian J Clin Biochem. 33 (2018) 382-386. 


\section{Table/Figure legends}

403

404 Table 1. Demographic characteristics of study's subjects.

405 Table 2. Histological classification and FIGO stages.

406 Abbreviations: FIGO, the International Federation of Gynecology and Obstetrics

407 Table 3. Values of CPH-I and ROMA of study's subjects

408 Table 4. The validity of $\mathrm{CPH}-\mathrm{I}$ and ROMA for the preoperative diagnosis of ovarian cancer 409 at the optimal cut-off.

410 Table 5. Diagnostic validity of $\mathrm{CPH}-\mathrm{I}$ and ROMA from literature.

411

412 Figure 1. Comparison of receiver operator characteristic curves for $\mathrm{CPH}-\mathrm{I}$ and $\mathrm{ROMA}$ in the 413 discrimination of benign tumors and borderline ovarian tumors (non-OC) from OC. 
Figures

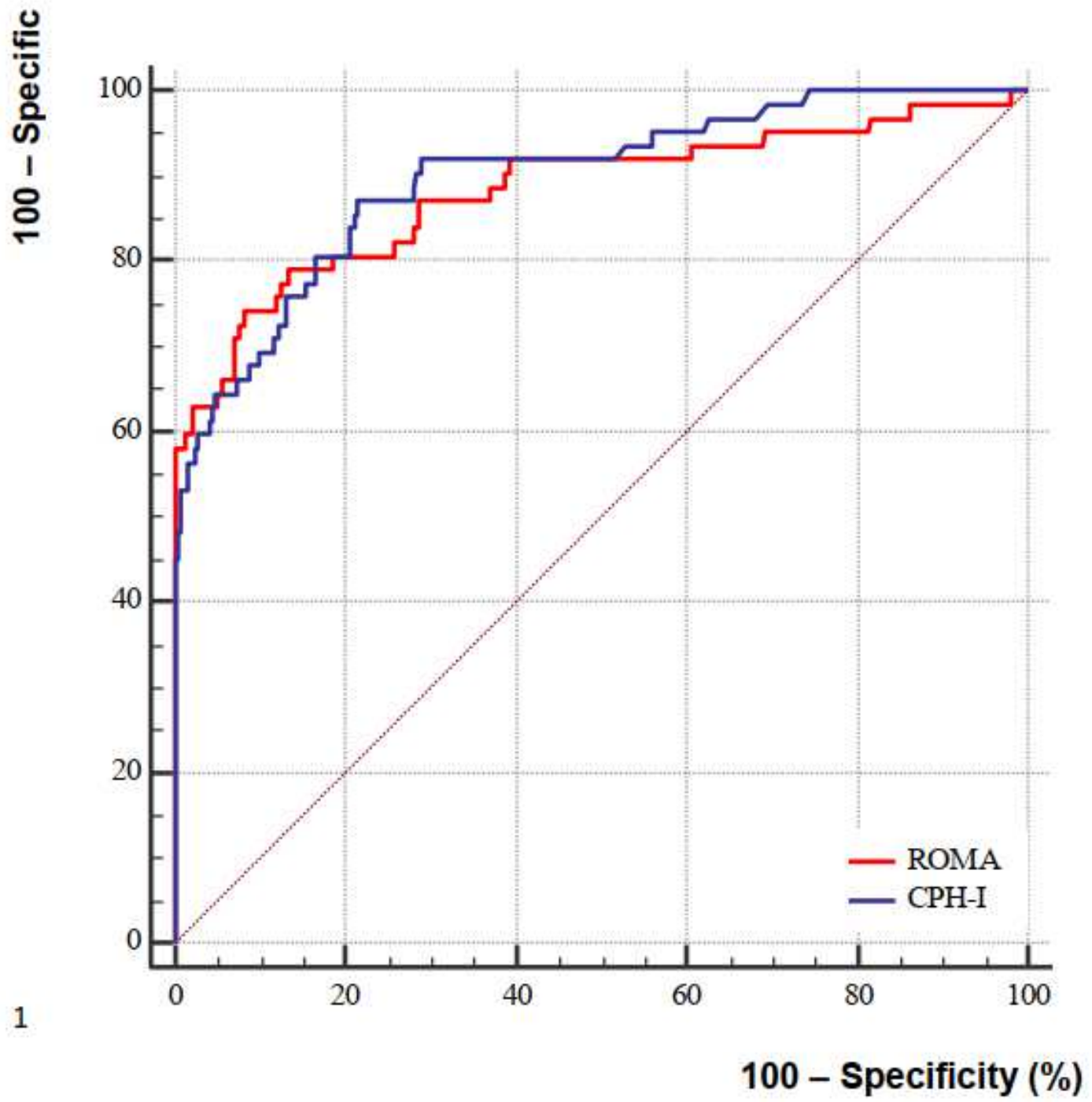

Graph 1. ROCs of CPH-I and ROMA values in study group.

\begin{tabular}{|c|c|c|c|c|}
\hline Index & $\begin{array}{l}\text { AUC } \\
(95 \% \mathrm{Cl})\end{array}$ & $\begin{array}{l}\mathrm{Se}(\%) \\
(95 \% \mathrm{Cl})\end{array}$ & $\begin{array}{l}\text { Sp (\%) } \\
(95 \% \mathrm{Cl})\end{array}$ & $p$-value \\
\hline $\mathrm{CPH}-\mathrm{I}$ & $\begin{array}{l}0,898 \\
(0,867-0,924)\end{array}$ & $\begin{array}{l}87.1 \\
(76.1-94.3)\end{array}$ & $\begin{array}{l}78.5 \\
(74.2-82.3)\end{array}$ & \multirow{2}{*}{0,4894} \\
\hline ROMA & $\begin{array}{l}0,882 \\
(0,849-0,909)\end{array}$ & $\begin{array}{l}74.2 \\
(61.5-84.5)\end{array}$ & $\begin{array}{l}91.8 \\
(88.7-94.2)\end{array}$ & \\
\hline
\end{tabular}

Figure 1

Comparison of receiver operator characteristic curves for $\mathrm{CPH}-\mathrm{I}$ and ROMA in the discrimination of benign tumors and borderline ovarian tumors (non-OC) from OC. 